\title{
BMJ Open Protocol for the WARM Hearts study: examining cardiovascular disease risk in middle-aged and older women - a prospective, observational cohort study
}

\author{
Alexandra V Rose (D) , ${ }^{1,2}$ Kevin F Boreskie (D) , ${ }^{1,2,3}$ Jacqueline L Hay, ${ }^{1,2}$ \\ Liam Thompson, ${ }^{1,2}$ Rakesh C Arora, ${ }^{2,4}$ Todd A Duhamel ${ }^{1,2}$
}

To cite: Rose AV, Boreskie KF, Hay JL, et al. Protocol for the WARM Hearts study: examining cardiovascular disease risk in middle-aged and older women - a prospective, observational cohort study. BMJ Open 2021;11:e044227. doi:10.1136/ bmjopen-2020-044227

- Prepublication history and additional supplemental material for this paper are available online. To view these files, please visit the journal online (http://dx.doi.org/10.1136/ bmjopen-2020-044227).

Received 08 September 2020 Accepted 11 May 2021

Check for updates

(C) Author(s) (or their employer(s)) 2021. Re-use permitted under CC BY-NC. No commercial re-use. See rights and permissions. Published by BMJ.

${ }^{1}$ Faculty of Kinesiology and Recreation Management, University of Manitoba, Winnipeg, Manitoba, Canada ${ }^{2}$ Institute of Cardiovascular Sciences, St.Boniface General Hospital Albrechtsen Research Centre, Winnipeg, Manitoba, Canada

${ }^{3}$ Max Rady College of Medicine, Rady Faculty of Health Sciences, University of Manitoba,

Winnipeg, Manitoba, Canada

${ }^{4}$ Department of Surgery, Max

Rady College of Medicine,

University of Manitoba,

Winnipeg, Manitoba, Canada

Correspondence to

Dr Todd A Duhamel;

TDuhamel@sbrc.ca

\section{ABSTRACT}

Introduction Cardiovascular disease (CVD) is a leading cause of death in women. Novel approaches to detect early signs of elevated CVD risk in women are needed. Enhancement of traditional CVD risk assessment approaches through the addition of procedures to assess physical function or frailty as well as novel biomarkers of cardiovascular, gut and muscle health could improve early identification. The Women's Advanced Risk-assessment in Manitoba (WARM) Hearts study will examine the use of novel non-invasive assessments and biomarkers to identify women who are at elevated risk for adverse cardiovascular events. Methods and analysis One thousand women 55 years of age or older will be recruited and screened by the WARM Hearts observational, cohort study. The two screening appointments will include assessments of medical history, gender variables, body composition, cognition, frailty status, functional fitness, physical activity levels, nutritional status, quality of life questionnaires, sleep behaviour, resting blood pressure (BP), BP response to moderate-intensity exercise, a non-invasive measure of arterial stiffness and heart rate variability. Blood sample analysis will be used to assess lipid and novel biomarker profiles and stool samples will support the characterisation of gut microbiota. The incidence of the adverse cardiovascular outcomes will be assessed 5 years after screening to compare WARM Hearts approaches to the Framingham Risk Score, the current clinical standard of assessing CVD risk in Canada.

Ethics and dissemination The University of Manitoba Health Research Ethics Board (7 October 2019) and the St Boniface Hospital Research Review Committee (7 October 2019) approved the trial (Ethics Number HS22576 (H2019:063)). Recruitment started 10 October 2020. Data gathered from the WARM Hearts study will be published in peer-reviewed journals and presented at national and international conferences. Knowledge translation strategies will be created to share our findings with stakeholders who are positioned to implement evidence-informed CVD risk assessment programming.

Trial registration number NCT03938155.

\section{INTRODUCTION}

Cardiovascular disease (CVD) is the leading cause of death for Canadian women. ${ }^{1}$ In the province of Manitoba, women develop heart
Strengths and limitations of this study

- The Women's Advanced Risk-assessment in Manitoba (WARM) Hearts study will identify adverse cardiovascular outcomes 5 years after screening using the personal health information number of each participant and the administrative records housed by the Manitoba Centre for Health Policy Population Health Research Data Repository.

- The WARM Hearts study will extensively characterise 1000 middle-aged and older women using traditional cardiovascular disease risk scores, as well as additional non-invasive techniques to assess physical function, frailty status, physical activity, sedentary and sleep behaviour patterns, blood pressure and arterial stiffness at rest and following moderate-intensity exercise, as well as biomarkers of cardiovascular, gut and muscle health.

- The WARM Hearts study will assess gender roles, relations and identity, which aligns with recommendations made by the Heart and Stroke Foundation of Canada to improve heart health research.

- The WARM Hearts study will use convenience sampling which has the inherent recruitment bias, in that participants who already may be active health consumers are more likely to participate.

disease approximately $10-15$ years later than men, with prevalence rates similar between sexes after 55 years of age. ${ }^{2}$ Even so, $78 \%$ of women who report to a hospital with symptoms go undetected for early signs of a myocardial infarction (MI). ${ }^{3}$ Additionally, Canadian women who have previously had an acute MI are more likely to die within a 1-year period as compared with men. ${ }^{45}$

Current efforts in Canada for identifying elevated risk for CVD (ie, 10-year estimate of risk for a future MI or stroke) focus on traditional risk factors such as age, sex, smoking, cholesterol and blood pressure (BP) ${ }^{6}$ using the Framingham Risk Score (FRS) ${ }^{7}$ However, the development of new methodologies are 
warranted as the FRS poorly discriminated between those who had onset CVD and those who did not over an 8-year follow-up. ${ }^{68}$ In fact, the absolute risk was markedly underestimated in women $(51 \%)$ versus men $(8 \%){ }^{68}$ There is inadequate evidence supporting sex and gender specific guidelines for cardiovascular care that results in women being under-represented, underdiagnosed and undertreated in the Canadian healthcare system. ${ }^{5}$

Implementing prognostic tools that examine parameters of physical function or frailty, novel cardiovascular function assessments, as well as novel biomarker analyses of cardiovascular, gut and muscle health may enhance CVD risk profiling. For example, a recent pooled analysis in a cohort of 154000 with a mean age of 71 years identified that frail older adults were at higher risk for CVD morbidity (Relative Risk (RR) 1.91, 95\% CI 1.85 to 2.08) and CVD mortality (HR 1.91, 95\% CI 1.77 to 2.05 ) over a median 3.2-year follow-up as compared with robust older adults. ${ }^{9}$ Novel blood-based biomarkers of CVD risk may also provide additional value to risk prediction by identifying pathophysiological processes earlier. ${ }^{10}$ For example, our previous work identified myokines that may act as biomarkers of frailty-related CVD progression. ${ }^{11}$ However, more evidence is needed to establish that parameters such as these have prognostic value in CVD risk profiling.

There is increasing evidence to suggest that several female-specific reproductive factors such as menstrual cycle, contraceptive use, fertility, pregnancy and menopause may be associated with the risk of CVD later in life. ${ }^{12}$ Several studies have reported that early menarche ${ }^{13}$ and early menopause ${ }^{14}$ are associated with elevated risk of CVD. A history of miscarriages and pregnancy complications has also been associated with elevated risk of CVD. ${ }^{15}{ }^{16}$ Exogenous oestrogen use, both contraceptives and postmenopausal hormone therapy, have been associated with elevated risk for CVD as well. ${ }^{15}$ Research is needed to establish if these relationships can provide useful prognostic value in CVD risk profiling.

The Women's Advanced Risk-assessment in Manitoba (WARM) Hearts study is a CVD screening programme adapted from the Assessment of Large and Small Artery Elasticity for the Early Detection of Cardiovascular Disease trial (NCT02863211). ${ }^{16}$ A broader number of non-invasive procedures are included to assess physical function, frailty status, physical activity, sedentary or sleep behaviour patterns and novel blood and stool-based biomarkers. Gender roles relations, and identity, as well as sex-specific risk factors will also be assessed to facilitate the future development of sex and gender-specific CVD risk profiles. Although the procedures in WARM Hearts were selected based on supporting evidence, it is not known if the approaches are feasible and have sufficient predictive value to implement within Canada. Therefore, the objective of this prospective, observational cohort is to: (1) determine if individual markers of CVD risk collected in the WARM Hearts cardiovascular screening protocol identify women 55 years of age and older who are at an elevated risk for experiencing adverse cardiovascular outcomes within a 5-year period after screening; (2) to create a novel CVD risk score for women incorporating the best markers of CVD risk collected in the WARM Hearts study; (3) compare the predictive ability of the WARM Hearts protocol and the FRS for predicting future adverse cardiovascular outcomes in women. We hypothesise that the WARM Hearts protocol will provide improved accuracy in identifying middle-aged and older women at an elevated risk for adverse cardiovascular outcomes in the 5-year period post-screening, as compared with the FRS.

\section{Potential impact of the study}

The WARM Hearts study may provide a novel CVD risk prognostic tool specifically for women. This prognostic tool could be considered for implementation in primary care settings if it demonstrates superior ability to screen women at elevated risk for future CVD compared with traditional methods.

\section{METHODS AND ANALYSIS \\ Study design}

The WARM Hearts protocol was developed based on the Standard Protocol Items: Recommendations for Intervention Trials guidelines. ${ }^{17}$ WARM Hearts publications will follow the Strengthening the Reporting of Observational Studies in Epidemiology ${ }^{18}$ and the Sex and Gender Equity in Research ${ }^{19}$ reporting guidelines. This research is using a prospective, observational design to determine if the use of novel CVD prognostic tools identifies women that are at high risk for experiencing an adverse cardiovascular event in the 5-year period after screening (figure 1). A description of the trial was registered on ClinicalTrials. gov.

\section{Patient and public involvement-patient engagement}

A formal participant engagement process was not used in the design of the trial. Patient partners will be sought to collaborate with the research team and stakeholders to summarise and disseminate the research findings to the community.

\section{Study setting}

The WARM Hearts study will be conducted in Winnipeg, Manitoba, Canada. Data collection appointments will be conducted at two sites: the University of Manitoba Active Living Centre and the St Boniface General Hospital Asper Clinical Research Institute.

\section{Recruitment}

One thousand women aged 55 years or older will be recruited through newspaper articles, an online webpage, media interviews, poster advertisement or presentations at community events to generate a convenience sample. Women who are interested to learn more about the research will be instructed to contact the WARM Hearts team by email or telephone. The research team will then provide an overview of the study and assess potential 


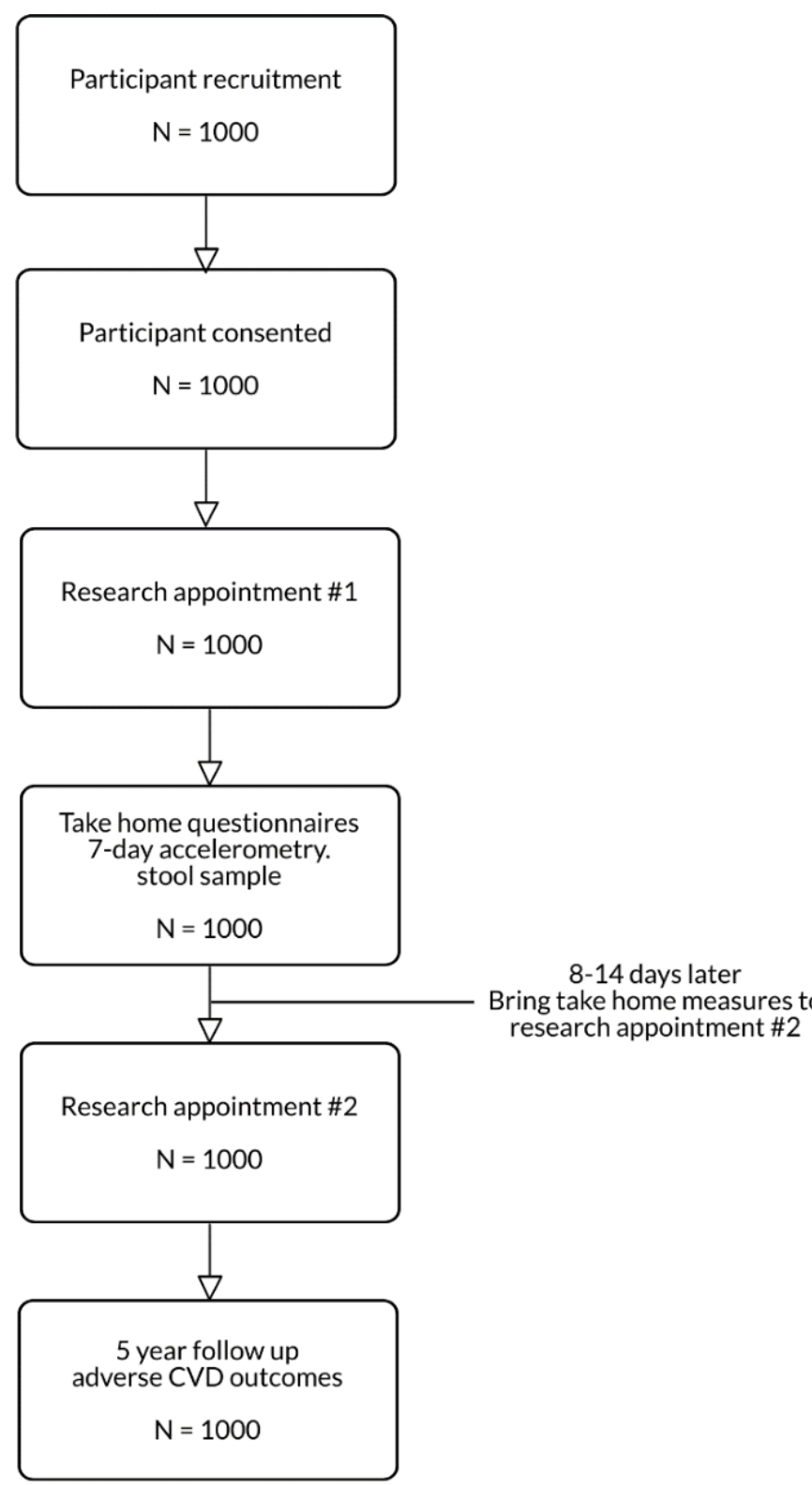

Figure 1 WARM Hearts flow chart. CVD, cardiovascular disease; WARM, Women's Advanced Risk-assessment in Manitoba.

participants for eligibility. Table 1 identifies the inclusion and exclusion criteria of the study.

\section{WARM HEARTS PROTOCOL Study procedures}

Eligible participants will be invited to attend two research appointments. The first appointment will be scheduled for $90 \mathrm{~min}$ at the University of Manitoba and used to secure written informed consent (see online supplemental files 1 and 2) and day 1 data collection. At the end of this first appointment, each participant will be provided an accelerometer, a stool sample collection kit and a take-home assessment package. The participants will be asked to complete these protocols on their own schedule at home. A second research appointment will then be scheduled
Table 1 Inclusion and exclusion criteria

\begin{tabular}{ll}
$\begin{array}{l}\text { Inclusion criteria } \\
\text { (both need to be fulfilled for } \\
\text { eligibility) }\end{array}$ & $\begin{array}{l}\text { Exclusion criteria } \\
\text { (any of the following result in } \\
\text { ineligibility) }\end{array}$ \\
\hline $\begin{array}{l}\text { (1) Women aged } 55 \text { and older } \\
\text { (2) Possess a Manitoba }\end{array}$ & $\begin{array}{l}\text { Previous hospitalisation or treatment } \\
\text { for: }\end{array}$ \\
$\begin{array}{l}\text { personal health information } \\
\text { number }\end{array}$ & (2) Acute myocardial infarction \\
& (3) Stroke/transient ischaemic attack \\
& (4) Percutaneous coronary \\
intervention \\
(5) Coronary artery bypass surgery \\
(6) Congestive heart failure \\
(7) Peripheral artery disease \\
(8) Congenital heart defects \\
(9) Arrhythmia \\
Additional exclusion: \\
(10) Medical advice against physical \\
activity \\
(11) Previous participant in the \\
Assessment of Large and Small \\
Artery Elasticity for the Early \\
Detection of Cardiovascular Disease \\
(NCTO2863211)
\end{tabular}

to occur 8-14days after the first appointment at the St Boniface Hospital Asper Clinical Research Institute. This approach enables the participants to return the accelerometer, stool sample and take-home assessment to the research team and to complete the day 2 data collection protocol, which requires approximately $45 \mathrm{~min}$ to complete. Table 2 summarises the data collected at each visit and in the take-home assessment.

Demographic, medical history and gender

Demographic and medical history data will be collected via questionnaire. Participants are asked about assigned sex at birth and their gender identity in line with recommendations by the Williams Institute ${ }^{20}$ and supported by the Canadian Institute for Health Research Institute of Gender and Health. ${ }^{21}$ Participants will also complete the validated gender-related score questionnaire ${ }^{22}$ previously used by the GENESIS-PRAXY investigators to examine gender as a continuous variable. ${ }^{23}$ Pelletier et al have previously used this tool to identify the association of gender with risk factors in patients with premature acute coronary syndrome. ${ }^{22}{ }^{23}$ Gender differences in CVD risk has been historically under examined. ${ }^{24}$

\section{Anthropometry and body composition}

Body composition impacts CVD risk factors ${ }^{25} 26$ and cardiovascular mortality. ${ }^{27}$ Participant height will be measured on a portable Seca stadiometer to the nearest $0.5 \mathrm{~cm}$. Waist circumference will be measured in accordance with the Canadian Society for Exercise Physiology (CSEP) guidelines at the height of the superior iliac crest. ${ }^{28} 29$ Body composition will be assessed through bioelectrical impedance analysis using the InBody 270.90 31 


\begin{tabular}{|c|c|}
\hline Variable & Instrument/questionnaire \\
\hline \multicolumn{2}{|c|}{ Day 1 (University of Manitoba Active Living Centre) } \\
\hline Consent process & $\begin{array}{l}\text { Protocol and biological sample consent } \\
\text { forms }\end{array}$ \\
\hline Demographic information & Questionnaire (age, sex, gender) ${ }^{9293}$ \\
\hline Medical history & $\begin{array}{l}\text { Questionnaire (medical history and } \\
\text { cardiovascular-related testing) }\end{array}$ \\
\hline Cognition & Montreal Cognitive Assessment ${ }^{37}$ \\
\hline Body composition & $\begin{array}{l}\text { Bioelectrical impedance analysis (InBody } \\
270)^{30} 31 \\
\text { Waist circumference } \\
\text { Body mass index }\end{array}$ \\
\hline Grip strength & Hand dynamometer \\
\hline 5-metre walk test & Gait speed test \\
\hline $\begin{array}{l}\text { Fried frailty phenotype } \\
\text { variables }\end{array}$ & $\begin{array}{l}\text { Questions about self-reported } \\
\text { exhaustion, unintentional weight loss. } \\
\text { Physical activity levels assessed by the } \\
\text { Paffenbarger Physical Activity Scale }{ }^{33}\end{array}$ \\
\hline Physical function & Senior Fitness Test ${ }^{40}$ \\
\hline Aerobic fitness & $\begin{array}{l}6 \text {-min walk test }{ }^{41} \text { with heart rate } \\
\text { variability assessed with heart } \\
\text { rate monitor and physical activity } \\
\text { characteristics assessed with } \\
\text { accelerometer }\end{array}$ \\
\hline
\end{tabular}

Personal health information number

\section{Take-home assessments ${ }^{\star}$}

\section{Demographic information Questionnaire}

Cardiovascular risk Questionnaire (smoking status, factors medications, history of cardiovascular disease)

Reproductive health

Questionnaire (menstrual history, contraception use, pregnancy history, menopause status, vasomotor symptoms, hormone therapy use)

\begin{tabular}{|c|c|}
\hline $\begin{array}{l}\text { Physical activity } \\
\text { characteristics }\end{array}$ & $\begin{array}{l}\text { Accelerometer worn for approximately } \\
1 \text { week }\end{array}$ \\
\hline Physical activity levels & $\begin{array}{l}\text { International Physical Activity } \\
\text { Questionnaire }^{67}\end{array}$ \\
\hline Gut microbiome & EasySampler stool sample kit \\
\hline Quality of life & $\begin{array}{l}\text { EUROQOL Five Dimension Five Level } \\
\text { and EQ-VAS }\end{array}$ \\
\hline Self-esteem & Self-Esteem Scale ${ }^{95}$ \\
\hline Gender information & $\begin{array}{l}\text { GENESIS-PRAXY Gender } \\
\text { Questionnaire }^{66}\end{array}$ \\
\hline Sleep quality & Pittsburgh Sleep Quality Index ${ }^{97}$ \\
\hline $\begin{array}{l}\text { Risk for impaired } \\
\text { nutritional states }\end{array}$ & SCREEN $\|^{77}$ \\
\hline Self-compassion & Self-compassion Scale ${ }^{98}$ \\
\hline Health anxiety & $\begin{array}{l}\text { Health anxiety subscale of the } \\
\text { Multidimensional Health Questionnaire }\end{array}$ \\
\hline \multicolumn{2}{|c|}{ Day 2 (Asper Clinical Research Institute) } \\
\hline \multicolumn{2}{|l|}{$\begin{array}{l}\text { Collect accelerometer } \\
\text { and stool samples }\end{array}$} \\
\hline Fasting blood sample & Phlebotomy \\
\hline
\end{tabular}

Continued

\begin{tabular}{|c|c|}
\hline Variable & Instrument/questionnaire \\
\hline Depression symptoms & Patient Health Questionnaire- $9^{100}$ \\
\hline Arterial stiffness & Mobil-O-Graph NG ${ }^{56}$ \\
\hline Resting blood pressure & Mobil-O-Graph NG ${ }^{56}$ \\
\hline $\begin{array}{l}\text { Blood pressure response } \\
\text { to moderate exercise }\end{array}$ & Mobil-O-Graph NG ${ }^{56}$ \\
\hline
\end{tabular}

${ }^{*}$ Completed on paper or via Research Electronic Data Capture System (REDCap) online survey.

EQ-VAS, EUROQOL Visual-Analogue Scale ; SCREEN II, Seniors in the Community: Risk Evaluation for Eating and Nutrition V.II .

\section{Frailty status}

Frailty will be assessed using two approaches. The Modified Fried Criteria (MFC) ${ }^{32}$ approach will use measures previously described by Fried et $a l^{33}$ along with measures of depression and cognitive impairment. Briefly, this phenotypic MFC approach classifies participants as frail if three or more of the following criteria are met: (1) unintentional weight loss, (2) self-reported exhaustion, (3) weakness, (4) slow walking speed, (5) low physical activity, (6) depression and (7) cognitive impairment. Exhaustion is assessed by the Center for Epidemiologic Studies - Depression Scale. ${ }^{34}$ Self-reported physical activity levels are assessed by the Paffenbarger Physical Activity Scale ${ }^{35}$ and unintentional weight loss is measured via a questionnaire. Gait speed is assessed by the 5 -metre walk test. ${ }^{36}$ Weakness is assessed by grip strength dynamometer. ${ }^{36}$ Cognitive impairment is assessed using the Montreal Cognitive Assessment. ${ }^{37}$

The second assessment of frailty will involve the creation of a frailty index (FI) using variables collected in WARM Hearts. This assessment methodology is based on frailty as an accumulation of deficits. ${ }^{38}$ The FI will be created according to guidelines described by Searle $e t$ $a l .{ }^{39}$ Briefly, the guidelines include: (1) deficits must have an association with health status; (2) deficit prevalence should increase with chronological age; (3) health deficits should not saturate too early; (4) deficits should cover a broad range of physiological systems; (5) frailty indices should be kept the same if planned to be used on the same cohort at multiple time points.

\section{Functional fitness assessments}

The Senior Fitness Test will be used to assess functional ability. ${ }^{40}$ This test measures upper and lower body strength through arm curls and the $30 \mathrm{~s}$ chair stand, respectively. The 8-foot up-and-go measures agility and dynamic balance. Upper body flexibility is measured by the backscratch test, while lower body flexibility is measured by the chair sit and reach. Each test has an associated risk zone threshold based on age and sex. Participants scoring below this threshold are considered at risk of loss for the associated functional ability. 


\section{6-min walk test}

Participants are instructed to walk as far as possible on a 172.5 metre track over a 6 min period. ${ }^{41}$ The distance covered during the 6-min walk test (6-MWT) test is recorded. Walk-based testing is an assessment of functional exercise capacity, ${ }^{42}$ which has been shown to influence risk of cardiovascular mortality in adult populations. ${ }^{43}$

\section{Heart rate variability}

Heart rate variability (HRV) will be recorded using a Polar Electro H7 heart rate monitor (Polar Electro Oy, Kempele, Finland) connected by bluetooth to an accelerometer. HRV measurements will be taken first with the participant in a seated position for $10 \mathrm{~min}$. Immediately following this static measurement, HRV will also be recorded during the 6-MWT. Kubios software ${ }^{44}$ will be used to clean the data, assess data quality and variables such as mean RR interval, SD of normal-normal intervals and root mean square of successive intervals.

\section{Blood sampling}

A blood sample will be collected to examine traditional CVD risk biomarkers for use in the FRS as well as novel biomarkers of cardiovascular and muscle health. Fifteen (15) $\mathrm{mL}$ of blood will be drawn by a phlebotomist and separated into four tubes: a serum silica tube $(1 \times 4 \mathrm{~mL})$, a lithium heparin tube $(1 \times 4 \mathrm{~mL})$ and two EDTA tubes $(2 \times 3 \mathrm{~mL})$. Two aliquots of whole blood from the serum silica tube will be snap frozen and stored at $-80^{\circ} \mathrm{C}$. After allowing the whole blood to coagulate and centrifuging at $2000 \times \mathrm{g}$ for $10 \mathrm{~min}$, two aliquots of serum will be collected, snap frozen and stored at $-80^{\circ} \mathrm{C}$. Blood samples in the EDTA and lithium heparin tubes will be centrifuged at $2000 \times \mathrm{g}$ for $10 \mathrm{~min}$ and then the plasma will be snap frozen and eight aliquots will be stored at $-80^{\circ} \mathrm{C}$. The planned blood analyses include: (1) total cholesterol, low-density lipoprotein cholesterol, high-density lipoprotein (HDL) cholesterol, triglycerides and fasting blood glucose using one of the lithium heparin plasma samples. The additional blood samples of serum, plasma and whole blood will be stored at $-80^{\circ} \mathrm{C}$ and saved for future analysis of novel biomarkers of CVD risk, such as myokines. ${ }^{11}$

\section{Stool sample collection}

Emerging evidence suggests a link between the gut microbiome and CVD progression. ${ }^{45-47}$ To characterise participant gut microbiome, stool samples will be collected using an EasySampler kit (American Laboratory Products Company, New Hampshire, USA). Participants will be provided the collection kit following the day 1 research appointment and instructed to collect a small sample in the provided container during the following 8-14 days. Participants will transport their stool sample with an ice pack and return it to the research team at the second appointment. The sample container will then be stored at $-80^{\circ} \mathrm{C}$ prior to being aliquoted and processed. Stool sample aliquots will be extracted from the sample container using a disposable biopsy punch (Miltex,
Integra LifeSciences, New Jersey, USA). One aliquot will be processed and stored in a ZR BashingBead Lysis Tube (Zymo Research, California, USA), and sent to the National Microbiology Laboratory (Winnipeg, Manitoba) to characterise gut microbiota via $16 \mathrm{~S}$ recombinant DNA sequencing. Three additional stool aliquots will be stored in a biobank at $-80^{\circ} \mathrm{C}$.

\section{Resting BP}

In accordance with Canadian Cardiovascular Society guidelines, ${ }^{48}$ the $\mathrm{BP}$ measurement will be made at the left upper arm while the participant is seated using a validated oscillometric device (Mobil-O-Graph). ${ }^{49}$ The Mobil-O-Graph ambulatory BP monitor is validated for the non-invasive assessment of systolic, diastolic and central $\mathrm{BP} .^{50}$ Central BP, an indirectly assessed measure of $\mathrm{BP}$ at the ascending aorta, is thought to potentially be a more important measure of $\mathrm{BP}$ as it better reflects the pressure exerted on target organs. ${ }^{151-53}$

For adults, a resting BP of $120 / 80 \mathrm{~mm} \mathrm{Hg}$ or less is considered normal, 120-139/80-89 mm Hg is considered prehypertensive, and greater than 140/90 mm Hg is classified as hypertension. ${ }^{49}$ A resting $\mathrm{BP}$ of $160 \mathrm{~mm}$ $\mathrm{Hg}$ systolic, $90 \mathrm{~mm} \mathrm{Hg}$ diastolic and a heart rate of 100 beats per minute are being used as screening cut-offs for proceeding with the data collection appointments as recommended by the CSEP guidelines. ${ }^{29}$ Participants whose values exceed any of these cut-offs are given $5 \mathrm{~min}$ to rest before the measurement is retaken. Scoring above the cut-off on two consecutive measurements terminates the appointment and participants are then recommended to visit their primary healthcare provider for an assessment. After visiting their primary healthcare provider, participants could then make an informed choice to reschedule their data collection appointment or to withdraw from the research.

\section{Non-invasive measure of arterial stiffness}

In addition to BP measurements, the Mobil-O-Graph assesses arterial stiffness. Using brachial oscillometry the Mobil-O-Graph calculates aortic pulse wave velocity (PWV) and additional central haemodynamic indices like augmentation index. PWV and the measures of central haemodynamics are estimated through analysis of the brachial cuff waveform and have acceptable accuracy as compared with intra-aortic readings ${ }^{54}$ and cardiac MRI. ${ }^{55}$ The Mobil-O-Graph has been validated in healthy individuals as well as patients with essential hypertension. ${ }^{5657}$ Arterial stiffness is associated with CVD risk and is predictive of CVD mortality in a variety of populations. ${ }^{58-62}$

\section{$\mathrm{BP}$ response to 3-min of exercise}

An exaggerated $\mathrm{BP}$ response to exercise may be an indicator for new-onset hypertension, ${ }^{63-65}$ CVD-related morbidity and mortality. ${ }^{63}$ Following the measurement of resting BP, the participant will complete $3 \mathrm{~min}$ of moderate-intensity exercise (ie, a five metabolic equivalent workload) on a treadmill. Immediately following the 
exercise protocol (within $30 \mathrm{~s}$ ), the participants will move to a seated position and have their BP measured again using the Mobil-O-Graph device. A normal BP response to moderate exercise is defined as a rise in systolic BP of under $30 \mathrm{~mm} \mathrm{Hg}$ and an absolute systolic BP of less than $169 \mathrm{~mm} \mathrm{Hg}$. A borderline response is defined as a rise of $30-39 \mathrm{~mm} \mathrm{Hg}$ and an absolute systolic BP of 170-179; whereas, an abnormal response is defined as a rise of $40 \mathrm{~mm} \mathrm{Hg}$ or more in systolic $\mathrm{BP}$ and an absolute systolic BP of $180 \mathrm{~mm} \mathrm{Hg}$ or higher. ${ }^{66}$ All participants with an abnormal $\mathrm{BP}$ response to $3 \mathrm{~min}$ of moderate exercise will be advised to speak with their primary healthcare provider.

\section{Questionnaires}

Participants will complete a series of questionnaires:

1. The International Physical Activity Questionnaire short version ${ }^{67}$ will be used to collect self-reported amount and intensity of physical activity completed in the last 7 days. Low levels of physical activity are linked to elevated CVD risk, ${ }^{68}$ CVD morbidity and CVD mortality. ${ }^{69}$

2. The EUROQOL Five Dimension Five Level will be used to evaluate the dimensions of mobility, self-care, usual activities, pain/discomfort and anxiety/depression. This validated questionnaire is routinely used for this purpose in healthy ${ }^{70}$ and a variety of disease populations. ${ }^{71-73}$ The EUROQOL Visual Analogue Scale will also be used, as it provides complementary information of the perceived health of the patient. ${ }^{74}$

3. Sleep quality and disturbances over a 1-month time interval are assessed using the Pittsburgh Sleep Quality Index. ${ }^{75}$ Previous research has suggested that abnormal sleep is associated with elevated risk for CVD. ${ }^{76}$

4. The Seniors in the Community: Risk Evaluation for Eating and Nutrition V.II will be used for the identification of risk for impaired nutritional status in community-living older adults. ${ }^{77}$ Diet can contribute to hypertension, diabetes, obesity and CVD. ${ }^{78}$

5. The Patient Health Questionnaire-9 (PHQ-9) will be used to screen for depressive symptoms. ${ }^{79}$ Depression is an independent risk factor for CVD ${ }^{80}$ PHQ-9 scores of 5, 10, 15 and 20 represent mild, moderate, moderately severe and severe depression, respectively. Participants with moderate to severe scores or whose response indicates thoughts of self-harm will be immediately directed to a healthcare provider. If the participant does not have access to a healthcare provider, they will be escorted to emergency care at the St Boniface General Hospital.

6. A questionnaire on menstrual history, contraception use, pregnancy history, menopause status, hormone therapy use will be used to characterise participant reproductive health. A more detailed version of the reproductive history questionnaire was added after September 2020, which included additional information on menstrual history, contraception use, pregnancy history, menopause status, vasomotor symptoms and hormone therapy use. Participants who were enrolled in the WARM Hearts study prior to September 2020 were given the opportunity to participate and consent into the WARM Hearts reproductive substudy to complete the more detailed version of the reproductive questionnaire. Out of the 240 participants who completed the WARM Hearts protocol prior to September 2020, a total of 225 participants consented to be contacted about future substudies. Of those who were eligible to be contacted, 203 participants have completed the survey as of 18 January 2020.

\section{Actigraphy}

ActiGraph wGT3X+ accelerometers worn on the nondominant wrist over a 7-day period will be used to measure physical activity, sedentary and sleep behaviour. ${ }^{81} 82$ The accelerometer has a dynamic range of $\pm 8 \mathrm{G}$ and will be sampled at rate of $100 \mathrm{~Hz}$. Accelerometer data will be downloaded using the ActiLife software proprietary to ActiGraph. Data will then be exported into nonproprietary CSV formats, such as in raw accelerations (g), counts per minute or vector magnitude epochs (range selected can be $1 \mathrm{~s}-60 \mathrm{~s}$ ). This flexible approach was chosen as there is no consensus on the gold standard for analysing sedentary behaviour, physical activity levels or sleep time. Analysis in the future will be done using both existing and emerging methodological approaches for these assessments. Parameters collected by the ActiGraph wGT3X+ accelerometerwill include raw acceleration, activity counts, steps taken, energy expenditures, body position and sleep/wake measurements. ${ }^{83}$ Low physical activity, ${ }^{68}$ sedentary ${ }^{84}$ and poor sleep behaviour ${ }^{85-87}$ are associated with CVD risk in adults.

\section{Framingham Risk Score}

A multivariate risk factor algorithm described by D'Agostino $e t a l^{88}$ is used to calculate the FRS for each participant. This tool is recommended for use in the Canadian clinical setting by the Canadian Cardiovascular Society. ${ }^{48}$ Age, sex, family CVD history and smoking status are collected by questionnaire; whereas, fasting glucose, HDL and total cholesterol measured from blood samples. BP is measured as already described using the Mobil-O-Graph.

\section{Adverse cardiovascular outcomes: 5-year follow-up}

Five years after screening, the incidence of adverse cardiovascular outcomes will be assessed in the cohort using Personal Health Information Numbers and the health administrative records housed by the Manitoba Centre for Health Policy (MCHP) Population Health Research Data Repository. De-identified data from the administrative records of Manitobans are stored in the repository with a corresponding scrambled identifier which allows the record to be linked in each of the data sets. Databases that will be accessed are outlined in table 3. Resources including hospital separation abstracts, medical claims and the Drug Program Information Network will provide information on medication usage, hospitalisations, diagnoses and procedures related to GVD. Vital statistics 
Table 3 Manitoba Centre for Health Policy data acquisition

\begin{tabular}{lll}
\hline Database & Data fields/variables & Rationale \\
\hline Manitoba Health Insurance Registry & $\begin{array}{l}\text { Estimated socioeconomic status and } \\
\text { information related to migration }\end{array}$ & $\begin{array}{l}\text { Socioeconomic status is an important health } \\
\text { determinant and migration away from Manitoba can be } \\
\text { identified }\end{array}$ \\
$\begin{array}{l}\text { Procedure codes on hospital } \\
\text { discharge abstracts }\end{array}$ & $\begin{array}{l}\text { Hospitalisation for cardiac diseases and } \\
\text { cardiac procedures performed }\end{array}$ & $\begin{array}{l}\text { Capture adverse cardiac events to test diagnostic } \\
\text { accuracy of WARM Hearts risk score variables versus } \\
\text { the Framingham Risk Score }\end{array}$ \\
Medical claims (physician billings) & $\begin{array}{l}\text { Diagnoses of cardiac diseases and } \\
\text { procedures performed }\end{array}$ & Track adverse cardiovascular events \\
Vital statistics & Cause of death & Track mortality rates \\
\hline Drug Program Information Network & Cardiac and other medications used & Track cardiovascular-related medication usage \\
\hline
\end{tabular}

WARM, Women's Advanced Risk-assessment in Manitoba.

will provide information on cause of death to allow for mortality rate tracking. The Manitoba Health Insurance Registry will be used for assessing socioeconomic status estimated based on postal code and for identifying participants that have migrated away from Manitoba during the follow-up period. We have defined adverse cardiovascular outcomes as hospitalisation or death due to ischaemic heart disease, acute myocardial infarction, angina, ischaemic stroke, haemorrhagic stroke, transient ischaemic attack, peripheral artery disease, congestive heart failure, cardiovascular disease-related interventions, such as coronary artery bypass graft or percutaneous coronary intervention or new-onset cases of hypertension. Adverse cardiovascular disease outcome definitions and codes

Table 4 Primary outcomes-adverse cardiovascular outcomes

\section{Adverse cardiovascular} outcomes

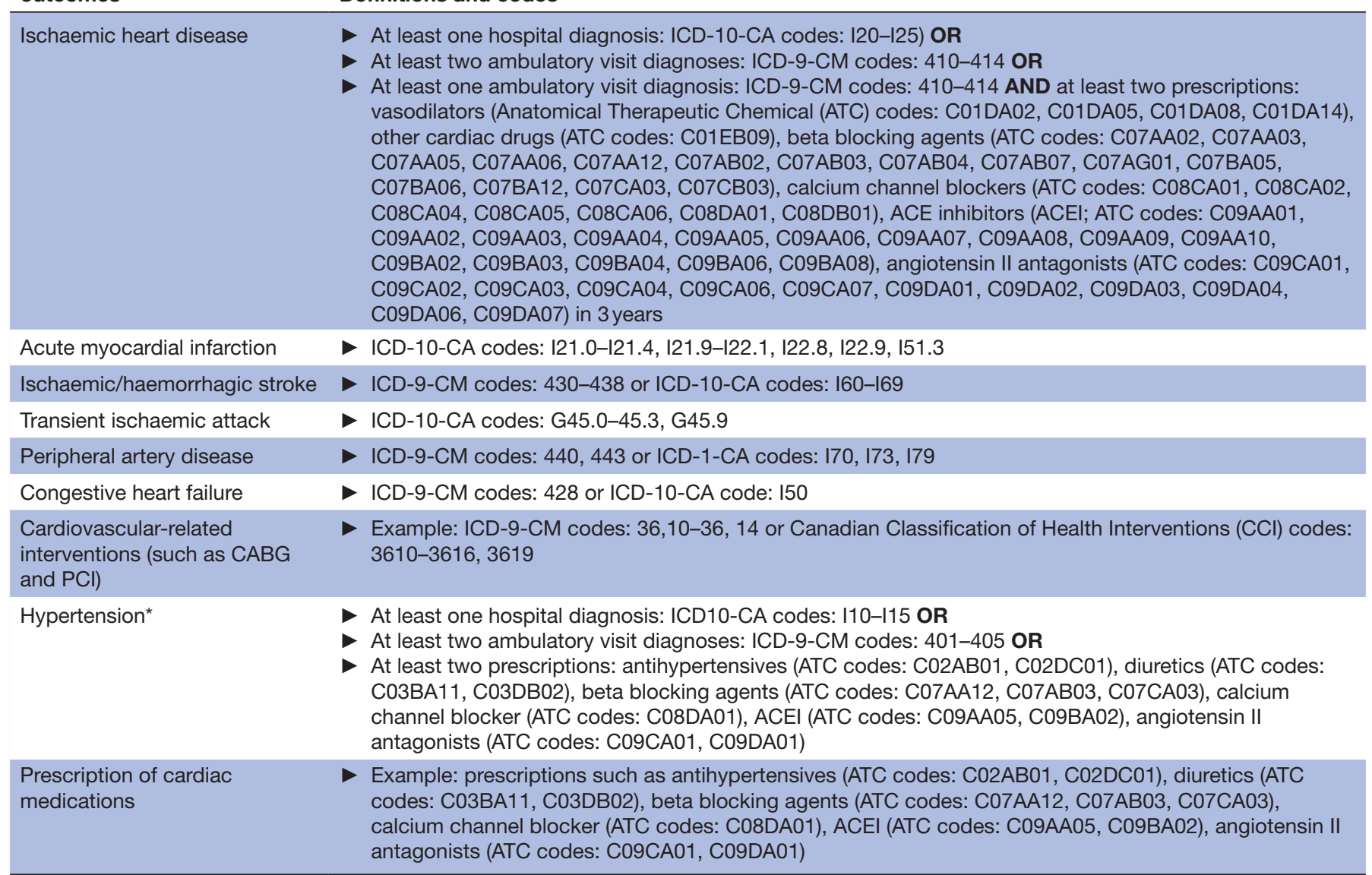

${ }^{*}$ This outcome will only be used for those participants with no prior diagnosis of hypertension.

CABG, coronary artery bypass graft; ICD-10-CA, International Classification of Diseases, 10th revision, Canada; ICD-9-CM, International

Classification of Diseases, 9th revision, clinical modification; $\mathrm{PCl}$, percutaneous coronary intervention. 
based on administrative data as previously used in the Manitoba context ${ }^{89}$ are listed in table 4 .

\section{Data management}

Initial WARM Hearts study data collection is expected to be complete in late 2021. De-identified data is expected to be available 6-months after that. Researchers wishing to access data should contact Dr Todd Duhamel (tduhamel@ sbrc.ca; orcid.org/0000-0001-8467-4800). A data dictionary will be available on request. An itemised list of the data being requested along with a brief proposal that outlines the rationale, hypotheses and proposed analyses will be required. Since biological samples represent a finite resource, the proposed analyses will be considered with regards to ethical approval, feasibility and novelty. A data sharing transfer agreement or a material transfer agreement must be signed and adhered to by collaborating parties and their respective institutions. Electronic data formats will be made available through the University of Manitoba Dataverse repository, following policies and procedures consistent with Tri-Council requirements.

\section{Statistical analysis}

Using estimations of adverse outcomes from published literature and data from our previous large cohort CVD screening project, ${ }^{16}$ we predict $187-241$ adverse health outcomes to occur in the 5-year period after the data collection in the cohort additional data informing this estimate are based on rates of ischaemic heart disease, acute MI, stroke, congestive heart failure, new prescriptions for cardiac medications (ACE inhibitors and statins) and new diagnoses of hypertension from the sex differences in health status, healthcare use and quality of care: A population-based analysis for Manitoba's Regional Health Authorities report ${ }^{90}$ and age-stratified data from the Chronic Disease and Injury Indicator Framework ${ }^{91}$ regression models will be used to identify variables that provide additional improvement of the model fit for predicting adverse cardiovascular events in a risk score at the end of the follow-up period. Two unique regression models will be developed to examine the association between the study variable of interest and adverse cardiovascular events. First, a conceptual model will be developed and consist of pre-selected hypothesised risk factors of interest for CVD as agreed on by the multidisciplinary research team following an extensive literature review. A second 'optimistic' model will be developed using a well-establish selection methodology such as a stepwise selection or hierarchal regression. The comparative predictability of the WARM Hearts CVD risk tool and the FRS will be assessed using survival analysis techniques, such as Kaplan-Meier methods, Cox proportional hazards model. Model discrimination will be assessed using techniques like receiver operating characteristic curves, Harrell's c-statistic and integrated discrimination improvement. The number of estimated CVD-related outcomes will allow us to conservatively use 10 variables in our model. Distribution and end results may dictate analyses used. The final prediction models will be externally validated through data collected in alternate cohort studies that include the same variables.

\section{Informed consent}

The University of Manitoba Health Research Ethics Board (REB; V.4.0 dated 7 October 2019) and the St Boniface Hospital Research Review Committee (V.4.0 dated 7 October 2019) approved the trial. Minor editorial revisions to the protocol documents were approved by the REB on 22 November 2019, 27 February 2020, 1 April 2020 and 16 June 2020. Individuals will be verbally informed of the study procedures by a research assistant and provided a copy of the patient information and consent forms. Each individual will have an adequate amount of time to consider their participation in the WARM Hearts study and will be given the opportunity to ask questions. After this process, if the individual decides to participate in the study, they will be asked to provide written consent on arrival at their first research appointment. Participants can decide not to participate in the study or to withdraw from the study at any time. Participants will be asked if they are willing to be contacted about future research studies.

\section{Trial monitoring and safety}

The research team is responsible for the study design, management, ethical conduct, analysis and dissemination of the WARM Hearts results. If a participant has a serious adverse event during the testing period, the protocol will be stopped. The participant will then be informed of the reasons for stopping the protocol. The appropriate steps will then be made to report the event to the University of Manitoba Health Research Ethics Board and the St Boniface Hospital Research Review Committee.

\section{End of study}

The WARM Hearts recruitment and CVD risk screening data collection phases of the research will end when 1000 participants have been successfully enrolled, which should be accomplished prior to 30 September 2021. Cross-sectional data analysis to characterise the cohort and examine associations between variables will be explored at this time. Adverse cardiovascular event data will be accessed through the MCHP 5 years after the completion of screening. Analyses of the adverse cardiovascular event data will then occur. Data will be published in peer-reviewed journals and presented at national and international conferences. Knowledge translation strategies and multimedia tools such as webinars, infographics and fact sheets will be created to share our findings with stakeholders who are positioned to design and implement evidence-informed CVD risk assessment approaches.

\section{Study status}

Recruitment for the WARM Hearts study began on 10 October 2019. Four hundred and eighty individuals were recruited to participate in the WARM Hearts trial as of 23 March 2020. Of these individuals, a total 
of 240 completed data collection. This date is relevant because it is when the University of Manitoba and St Boniface Hospital suspended research activities with human participants due to the COVID-19 public health pandemic. Recruitment and data collection will recommence when public health officials in Manitoba and the institutions deem it is appropriate to relaunch the research. We anticipate the cohort recruitment will be completed in late 2021.

\section{ETHICS AND DISSEMINATION}

The University of Manitoba Health Research Ethics Board (7 October 2019) and the St Boniface Hospital Research Review Committee (7 October 2019) approved the trial (Ethics Number HS22576 (H2019:063)). Recruitment started 10 October 2020. Data gathered from the WARM Hearts study will be published in peer-reviewed journals and presented at national and international conferences. Knowledge translation strategies will be created to share our findings with stakeholders who are positioned to implement evidence-informed CVD risk assessment programming.

\section{Twitter Kevin F Boreskie @kboreskie and Rakesh C Arora @TheRakeshArora}

Contributors TAD, KFB and JLH were involved in conception and design of research. TAD secured funding for the project. KFB, JLH, RCA and TAD obtained ethics approval. AVR, KFB, JLH and LT drafted the manuscript. All authors edited and revised the manuscript. All authors approved the final version of the manuscript. AVR, LT and research staff are assisting with data collection. TAD, KFB and JLH are managing the research. AVR and research staff are assisting with administration support.

Funding Operating funds to support this research are generously provided by the St Boniface Hospital Research Foundation. Infrastructure supports are provided by the University of Manitoba, the St Boniface General Hospital Albrechtsen Research Centre and the I.H. Asper Clinical Research Institute. KFB is supported by a Frederick Banting and Charles Best Canada Graduate Scholarship - Canadian Institute for Health Research (CIHR) Doctoral Award. JLH is supported by CIHR Vanier Scholarship. AVR is supported by a Faculty of Kinesiology and Recreation Management Graduate Scholarship through the University of Manitoba.

\section{Competing interests None declared.}

Patient consent for publication Not required.

Provenance and peer review Not commissioned; externally peer reviewed.

Supplemental material This content has been supplied by the author(s). It has not been vetted by BMJ Publishing Group Limited (BMJ) and may not have been peer-reviewed. Any opinions or recommendations discussed are solely those of the author(s) and are not endorsed by BMJ. BMJ disclaims all liability and responsibility arising from any reliance placed on the content. Where the content includes any translated material, BMJ does not warrant the accuracy and reliability of the translations (including but not limited to local regulations, clinical guidelines, terminology, drug names and drug dosages), and is not responsible for any error and/or omissions arising from translation and adaptation or otherwise.

Open access This is an open access article distributed in accordance with the Creative Commons Attribution Non Commercial (CC BY-NC 4.0) license, which permits others to distribute, remix, adapt, build upon this work non-commercially, and license their derivative works on different terms, provided the original work is properly cited, appropriate credit is given, any changes made indicated, and the use is non-commercial. See: http://creativecommons.org/licenses/by-nc/4.0/.

\section{ORCID iDs}

Alexandra V Rose http://orcid.org/0000-0003-4600-2848

Kevin F Boreskie http://orcid.org/0000-0002-9763-2762
REFERENCES

1 GBD 2016 Causes of Death Collaborators. Global, regional, and national age-sex specific mortality for 264 causes of death, 19802016: a systematic analysis for the global burden of disease study 2016. Lancet 2017;390:1151-210.

2 Kettner J. Chief provincial public health officer's report on the health status of manitobans 2010 priorities for prevention: everyone, every place, every day. 272, 2010.

3 Heart \& Stroke 2018 Heart Report. Ms Understood Women's hearts are victims of a system that is ill-equipped to diagnose, treat and support them, 2018.

4 Sedlak TL, Lee M, Izadnegahdar M, et al. Sex differences in clinical outcomes in patients with stable angina and NO obstructive coronary artery disease. Am Heart J 2013;166:38-44.

5 Norris CM, Yip CYY, Nerenberg KA, et al. State of the science in women's cardiovascular disease: a Canadian perspective on the influence of sex and gender. J Am Heart Assoc 2020;9:e015634.

6 Rodondi N, Locatelli I, Aujesky D, et al. Framingham risk score and alternatives for prediction of coronary heart disease in older adults. PLoS One 2012;7:e34287.

7 Shlipak MG, Elmouchi DA, Herrington DM, et al. The incidence of unrecognized myocardial infarction in women with coronary heart disease. Ann Intern Med 2001;134:1043-7.

8 O'Callaghan KM. Solutions for disparities for women with heart disease. J Cardiovasc Transl Res 2009;2:518-25.

9 Farooqi MAM, Gerstein H, Yusuf S, et al. Accumulation of Deficits as a Key Risk Factor for Cardiovascular Morbidity and Mortality: A Pooled Analysis of 154000 Individuals. J Am Heart Assoc 2020;9:e014686.

10 Wang J, Tan G-J, Han L-N, et al. Novel biomarkers for cardiovascular risk prediction. J Geriatr Cardiol 2017;14:135-50.

11 Boreskie KF, Oldfield CJ, Hay JL, et al. Myokines as biomarkers of frailty and cardiovascular disease risk in females. Exp Gerontol 2020;133:1-7.

12 Merritt MA, Riboli E, Murphy N, et al. Reproductive factors and risk of mortality in the European prospective investigation into cancer and nutrition; a cohort study. BMC Med 2015;13:252.

13 Canoy D, Beral V, Balkwill A, et al. Age at menarche and risk of coronary heart disease in the UK Million women study. Int $J$ Epidemiol 2015;44:i52-3.

14 Atsma F, Bartelink M-LEL, Grobbee DE, et al. Postmenopausal status and early menopause as independent risk factors for cardiovascular disease: a meta-analysis. Menopause 2006;13:265-79.

15 Lawlor DA, Emberson JR, Ebrahim S, et al. Is the association between parity and coronary heart disease due to biological effects of pregnancy or adverse lifestyle risk factors associated with childrearing? findings from the British women's heart and health study and the British regional heart study. Circulation 2003;107:1260-4.

16 Boreskie KF, Kehler DS, Costa EC, et al. Protocol for the happy hearts study: cardiovascular screening for the early detection of future adverse cardiovascular outcomes in middle-aged and older women: a prospective, observational cohort study. BMJ Open 2017;7:1-9.

17 Chan A-W, Tetzlaff JM, Gøtzsche PC, et al. Spirit 2013 explanation and elaboration: guidance for protocols of clinical trials. BMJ 2013;346:1-42.

18 von Elm E, Altman DG, Egger M, et al. The strengthening the reporting of observational studies in epidemiology (STROBE) statement: guidelines for reporting observational studies. Int J Surg 2014;12:1495-9.

19 Heidari S, Babor TF, De Castro P, et al. Sex and gender equity in research: rationale for the SAGER guidelines and recommended use. Res Integr Peer Rev 2016;1:2.

20 Park A. Reachable : Data collection methods for sexual orientation and gender identity. . The Williams Institue, 2016: 2016. 1-8.

21 Bauer G, Title N. Canadian Institutes of health research, 2012.

22 Pelletier R, Ditto B, Pilote L. A composite measure of gender and its association with risk factors in patients with premature acute coronary syndrome. Psychosom Med 2015;77:517-26.

23 Pelletier R, Khan NA, Cox J, et al. Sex versus gender-related characteristics: which predicts outcome after acute coronary syndrome in the young? J Am Coll Cardiol 2016;67:127-35.

24 O'Neil A, Scovelle AJ, Milner AJ, et al. Gender/sex as a social determinant of cardiovascular risk. Circulation 2018;137:854-64.

25 Hernandez-Martinez A, Martinez-Rosales E, Alcaraz-lbañez M, et al. Influence of body composition on arterial stiffness in middle-aged adults: healthy UAL cross-sectional study. Medicina 2019;55. doi:10.3390/medicina55070334. [Epub ahead of print: 03 Jul 2019].

26 Ittermann T, Werner N, Lieb W, et al. Changes in fat mass and fat-free-mass are associated with incident hypertension 
in four population-based studies from Germany. Int $J$ Cardiol 2019;274:372-7.

27 Srikanthan $\mathrm{P}$, Horwich TB, Tseng $\mathrm{CH}$. Relation of muscle mass and fat mass to cardiovascular disease mortality. Am J Cardiol 2016;117:1355-60.

28 Janssen I, Katzmarzyk PT, Ross R. Waist circumference and not body mass index explains obesity-related health risk. Am J Clin Nutr 2004;79:379-84

29 Canadian Society of Exercise Physiology. CSEP certified personal trainer $₫$ update to Pre-Participation screening procedures CSEP certified personal trainer ${ }^{\circledR}, 2017$ : 2-4

30 Ling CHY, de Craen AJM, Slagboom PE, et al. Accuracy of direct segmental Multi-frequency bioimpedance analysis in the assessment of total body and segmental body composition in middle-aged adult population. Clin Nutr 2011;30:610-5.

31 Sato S, Demura S, Kitabayashi T, et al. Segmental body composition assessment for obese Japanese adults by singlefrequency bioelectrical impedance analysis with 8-point contact electrodes. J Physiol Anthropol 2007;26:533-40.

32 Rothman MD, Leo-Summers L, Gill TM. Prognostic significance of potential frailty criteria. J Am Geriatr Soc 2008;56:2211-6.

33 Fried LP, Tangen CM, Walston J, et al. Frailty in older adults: evidence for a phenotype. J Gerontol A Biol Sci Med Sci 2001;56:M146-57.

34 Radloff LS. The CES-D scale: a self-report depression scale for research in the general population. Appl Psychol Meas 1977:1:385-401.

35 Nowak Z, Plewa M, Skowron M, et al. Paffenbarger physical activity questionnaire as an additional tool in clinical assessment of patients with coronary artery disease treated with angioplasty. Kardiol Pol 2010;68:32-9.

36 Clegg A, Young J, lliffe S, et al. Frailty in elderly people. Lancet 2013;381:752-62.

37 Nasreddine ZS, Phillips NA, Bédirian V, et al. The Montreal cognitive assessment, MoCA: a brief screening tool for mild cognitive impairment. J Am Geriatr Soc 2005;53:695-9.

38 Mitnitski AB, Mogilner AJ, Rockwood K. Accumulation of deficits as a proxy measure of aging. ScientificWorldJournal 2001;1:323-36.

39 Searle SD, Mitnitski A, Gahbauer EA, et al. A standard procedure for creating a frailty index. BMC Geriatr 2008;8:24.

40 Rikli RE, Jones CJ. Development and validation of a functional fitness test for Community-Residing older adults. J Aging Phys Act 1999;7:129-61.

41 Beatty AL, Schiller NB, Whooley MA. Six-minute walk test as a prognostic tool in stable coronary heart disease: data from the heart and soul study. Arch Intern Med 2012;172:1096-102.

42 Fleg JL, Piña IL, Balady GJ, Chaitman BR, Fletcher B, et al. Assessment of functional capacity in clinical and research applications: an Advisory from the Committee on exercise, rehabilitation, and prevention, Council on clinical cardiology, American heart association. Circulation 2000;102:1591-7.

43 Redberg RF, Whiteman MK, a FJ, et al. And all-cause death in asymptomatic women. Methods 2007;290:1600-7.

44 Tarvainen MP, Niskanen J-P, Lipponen JA, et al. Kubios HRV-heart rate variability analysis software. Comput Methods Programs Biomed 2014;113:210-20.

45 Tang WHW, Hazen SL. The gut microbiome and its role in cardiovascular diseases. Circulation 2017;135:1008-10.

46 Ahmad AF, Dwivedi G, O'Gara F, O'Gara F, et al. The gut microbiome and cardiovascular disease: current knowledge and clinical potential. Am J Physiol Heart Circ Physiol 2019;317:H923-38.

47 Lau K, Srivatsav V, Rizwan A, et al. Bridging the gap between gut microbial dysbiosis and cardiovascular diseases. Nutrients 2017;9. doi:10.3390/nu9080859. [Epub ahead of print: 10 Aug 2017].

48 Anderson TJ, Grégoire J, Pearson GJ, et al. Canadian cardiovascular Society guidelines for the management of dyslipidemia for the prevention of cardiovascular disease in the adult. Can J Cardiol 2016;2016:1263-82.

49 Nerenberg KA, Zarnke KB, Leung AA, et al. Hypertension Canada's 2018 guidelines for diagnosis, risk assessment, prevention, and treatment of hypertension in adults and children. Can J Cardiol 2018;34:506-25.

50 Jones CR, Taylor K, Chowienczyk P, et al. A validation of the Mobil O graph (version 12) ambulatory blood pressure monitor. Blood Press Monit 2000:5:233-8.

51 Agabiti-Rosei E, Mancia G, O'Rourke MF, et al. Central blood pressure measurements and antihypertensive therapy: a consensus document. Hypertension 2007;50:154-60.
52 Waddell TK, Dart AM, Medley TL, et al. Carotid pressure is a better predictor of coronary artery disease severity than brachial pressure. Hypertension 2001;38:927-31.

53 Roman MJ, Devereux RB, Kizer JR, et al. Central pressure more strongly relates to vascular disease and outcome than does brachial pressure: the strong heart study. Hypertension 2007;50:197-203.

54 Hametner B, Wassertheurer S, Kropf J, et al. Oscillometric estimation of aortic pulse wave velocity: comparison with intraaortic catheter measurements. Blood Press Monit 2013;18:173-6.

55 Feistritzer H-J, Reinstadler SJ, Klug G, et al. Comparison of an oscillometric method with cardiac magnetic resonance for the analysis of aortic pulse wave velocity. PLoS One 2015;10:1-12.

56 Weiss W, Gohlisch C, Harsch-Gladisch C, et al. Oscillometric estimation of central blood pressure: validation of the Mobil-OGraph in comparison with the SphygmoCor device. Blood Press Monit 2012;17:128-31.

57 Wassertheurer S, Kropf J, Weber T, et al. A new oscillometric method for pulse wave analysis: comparison with a common tonometric method. J Hum Hypertens 2010;24:498-504.

58 Shoji T, Maekawa K, Emoto M, et al. Arterial stiffness predicts cardiovascular death independent of arterial thickness in a cohort of hemodialysis patients. Atherosclerosis 2010;210:145-9.

59 Pereira T, Maldonado J, Pereira L, et al. Aortic stiffness is an independent predictor of stroke in hypertensive patients. Arq Bras Cardiol 2013;100:437-43.

60 Choi CU, Park EB, Suh SY, et al. Impact of aortic stiffness on cardiovascular disease in patients with chest pain: assessment with direct intra-arterial measurement. Am J Hypertens 2007;20:1163-9.

61 Cruickshank K, Riste L, Anderson SG, et al. Aortic pulse-wave velocity and its relationship to mortality in diabetes and glucose intolerance: an integrated index of vascular function? Circulation 2002;106:2085-90.

62 Mattace-Raso FUS, van der Cammen TJM, Hofman A, et al. Arterial stiffness and risk of coronary heart disease and stroke: the Rotterdam study. Circulation 2006;113:657-63.

$63 \mathrm{Kim} \mathrm{D,} \mathrm{Ha} \mathrm{J-W.} \mathrm{Hypertensive} \mathrm{response} \mathrm{to} \mathrm{exercise:} \mathrm{mechanisms}$ and clinical implication. Clin Hypertens 2016;22:16-19.

64 Schultz MG, Hare JL, Marwick TH, et al. Masked hypertension is "unmasked" by low-intensity exercise blood pressure. Blood Press 2011;20:284-9.

65 Singh JP, Larson MG, Manolio TA, et al. Blood pressure response during treadmill testing as a risk factor for new-onset hypertension. The Framingham heart study. Circulation 1999;99:1831-6.

66 Duprez DA, Florea N, Zhong W, et al. Vascular and cardiac functional and structural screening to identify risk of future morbid events: preliminary observations. J Am Soc Hypertens 2011;5:401-9.

67 Craig CL, Marshall AL, Sjöström M, et al. International physical activity questionnaire: 12 -country reliability and validity. Med Sci Sports Exerc 2003;35:1381-95.

68 Oguma Y, Shinoda-Tagawa T. Physical activity decreases cardiovascular disease risk in women: review and meta-analysis. Am J Prev Med 2004;26:407-18.

69 Rodriguez BL, Curb JD, Burchfiel CM, et al. Physical activity and 23-year incidence of coronary heart disease morbidity and mortality among middle-aged men. The Honolulu heart program. Circulation 1994;89:2540-4.

$70 \mathrm{Kim}$ TH, Jo M-W, Lee S-il, et al. Psychometric properties of the EQ-5D-5L in the general population of South Korea. Qual Life Res 2013;22:2245-53

71 Koh D, Abdullah AMKbin, Wang P, et al. Validation of Brunei's Malay EQ-5D questionnaire in patients with type 2 diabetes. PLoS One 2016;11:e0165555-9.

72 Dyer MTD, Goldsmith KA, Sharples LS, et al. A review of health utilities using the EQ-5D in studies of cardiovascular disease. Health Qual Life Outcomes 2010;8:13-12.

73 Nolan CM, Longworth L, Lord J, et al. The EQ-5D-5L health status questionnaire in COPD: validity, responsiveness and minimum important difference. Thorax 2016;71:493-500.

74 Feng Y, Parkin D, Devlin NJ. Assessing the performance of the EQVAS in the NHS PROMs programme. Qual Life Res 2014;23:977-89.

75 Buysse DJ, Reynolds CF, Monk TH, et al. The Pittsburgh sleep quality index: a new instrument for psychiatric practice and research. Psychiatry Res 1989;28:193-213.

76 Kwok CS, Kontopantelis E, Kuligowski G, et al. Self-reported sleep duration and quality and cardiovascular disease and mortality: a dose-response meta-analysis. J Am Heart Assoc 2018;7:e008552.

77 Keller HH, Goy R, Kane S-L. Validity and reliability of screen II (seniors in the community: risk evaluation for eating and nutrition, version II). Eur J Clin Nutr 2005;59:1149-57. 
78 Lim SS, Vos T, Flaxman AD, et al. A comparative risk assessment of burden of disease and injury attributable to 67 risk factors and risk factor clusters in 21 regions, 1990-2010: a systematic analysis for the global burden of disease study 2010. Lancet 2012;380:2224-60.

79 Kroenke K, Spitzer RL, Williams JBW. The PHQ-9. J Gen Intern Med 2001;16:606-13.

80 Blumenthal JA. Depression and coronary heart disease: association and implications for treatment. Cleve Clin J Med 2008;75 Suppl 2:48-53.

81 Doherty A, Jackson D, Hammerla N, et al. Large scale population assessment of physical activity using wrist worn accelerometers: the UK Biobank study. PLoS One 2017;12:1-14.

82 Full KM, Kerr J, Grandner MA, et al. Validation of a physical activity accelerometer device worn on the hip and wrist against polysomnography. Sleep Health 2018;4:209-16.

83 Actigraph L. Activity monitor comparison, 2020. Available: https:// www.actigraphcorp.com/activity-monitor-comparison/

84 Hajduk AM, Chaudhry SI. Sedentary behavior and cardiovascular risk in older adults: a scoping review. Curr Cardiovasc Risk Rep 2016;10. doi:10.1007/s12170-016-0485-6. [Epub ahead of print: 29 $012016]$.

85 Bertisch SM, Pollock BD, Mittleman MA, et al. Insomnia with objective short sleep duration and risk of incident cardiovascular disease and all-cause mortality: sleep heart health study. Sleep 2018;41:1-9.

86 Gottlieb DJ, Redline S, Nieto FJ, et al. Association of usual sleep duration with hypertension: the sleep heart health study. Sleep 2006;29:1009-14.

87 Wolk R, Gami AS, Garcia-Touchard A, et al. Sleep and cardiovascular disease. Curr Probl Cardiol 2005;30:625-62.

88 D'Agostino RB, Vasan RS, Pencina MJ, et al. General cardiovascular risk profile for use in primary care: the Framingham heart study. Circulation 2008;117:743-53.

89 Alan K, Patricia M, Dan C. Understanding the health system use of ambulatory care patients. Winnipeg: Manitoba Centre for Health Policy, University of Manitoba, 2013.
90 Randy F, Patricia M. Sex differences in health status, health care use, and quality of care: a population-based analysis for manitoba's regional health authorities. Winnipeg: Manitoba Centre for Health Policy, 2005.

91 Surveillance and Epidemiology Division, Centre for Chronic Disease Prevention, Public Health Agency of Canada. Chronic disease and injury indicator framework: quick STATs, fall 2014 edition. Chronic Dis Inj Can 2014;34:272-5.

92 Bauer G. Making sure everyone counts: considerations for inclusion, identification and analysis of transgender and transsexual participants in health surverys. CIHR Inst Gend Heal - Webinar Ser Integr Gend Sex Heal Res, 2012. Available: http://hdl.handle.net/ 2429/41729

93 Tate CC, Ledbetter JN, Youssef CP. A two-question method for assessing gender categories in the social and medical sciences. $J$ Sex Res 2013;50:767-76.

94 Herdman M, Gudex C, Lloyd A, et al. Development and preliminary testing of the new five-level version of EQ-5D (EQ-5D-5L). Qual Life Res 2011;20:1727-36.

95 Rosenberg M. Society and the adolescent Self-Image. Am Assoc Adv Sci 1965;148:804.

96 Pilote L, Karp I. GENESIS-PRAXY (gender and sex determinants of cardiovascular disease: from bench to beyond-Premature acute coronary syndrome). Am Heart J 2012;163:741-6.

97 Beaudreau SA, Spira AP, Stewart A, et al. Validation of the Pittsburgh sleep quality index and the Epworth Sleepiness scale in older black and white women. Sleep Med 2012;13:36-42.

98 NEFF KD. The development and validation of a scale to measure Self-Compassion. Self and Identity 2003;2:223-50.

99 SW JG. The multidimensional health questionnaire. Am J Health Behav 1996;21:33-42.

100 Kroenke K, Spitzer RL, Williams JB. The PHQ-9: validity of a brief depression severity measure. J Gen Intern Med 2001;16:606-13. 